\title{
基于生成对抗网络的多聚焦图像融合
}

\author{
蒋留兵 ${ }^{1)}$, 张点 ${ }^{2}{ }^{*}$, 潘 $^{(2)}$, 郑朋 $^{2)}$, 车例 ${ }^{1)}$ \\ ${ }^{1)}$ (桂林电子科技大学信息与通信学院 桂林 541004) \\ ${ }^{2)}$ (桂林电子科技大学计算机与信息安全学院 桂林 541004) \\ (albertzhang00000@sina.com)
}

\begin{abstract}
摘 要: 多聚焦图像融合能够融合同一场景下具有不同聚焦部分的一系列图像. 为了克服多聚焦图像融合模糊特征 提取中存在的不足，提出一种基于 U-Net 的生成对抗网络模型. 首先，生成器采用 U-Net 和 SSE 对多聚焦图像的特征 进行提取, 并完成图像融合; 其次, 判别器采用卷积层对已知的融合结果和生成器生成的融合图像进行分辨; 然后, 损失函数采用生成器的对抗损失、映射损失、梯度损失、均方误差损失和判别器对抗损失对生成网络进行参数调节; 最后, 将生成器、判别器和损失函数组成生成对抗网络模型, 并进行实验. Pascal VOC2012 数据集作为生成对抗网络 的训练集，包括近焦图像、远焦图像、映射图像和融合图像. 实验结果证明，该生成对抗网络模型能够有效地提取多 聚焦图像中的模糊特征，且融合图像在互信息、相位一致性和感知相似性等方面表现优异.
\end{abstract}

关键词: 多聚焦图像融合; U-Net; 生成对抗网络; 损失函数

中图法分类号: TP391.41 DOI: 10.3724/SP.J.1089.2021.18770

\section{Multi-Focus Image Fusion Based on Generative Adversarial Network}

\author{
Jiang Liubing ${ }^{1)}$, Zhang $\operatorname{Dian}^{2)^{*}}$, Pan $\mathrm{Bo}^{2)}$, Zheng Peng ${ }^{2)}$, and $\mathrm{Che} \mathrm{Li}^{1)}$ \\ 1) (School of Information and Communication, Guilin University of Electronic Technology, Guilin 541004) \\ ${ }^{2)}$ (School of Computer Science and Information Security, Guilin University of Electronic Technology, Guilin 541004)
}

\begin{abstract}
Multi-focus image fusion can fuse a series of images that have a different focus in the same scene. To overcome the disadvantage extraction of the blurring character in multi-focus images, a generative adversarial network model based on U-Net is proposed. Firstly, the generator uses U-Net and SSE to extract the feature of the multi-focus image, and fuses images. Then, the discriminator uses convolutional layers to distinguish the fused result between the existed and the generative. Furthermore, a loss function has the loss of adversarial in the generator, loss of mapping, loss of gradient, loss mean square error and the loss of adversarial in the discriminator. The train data of generative adversarial network uses the dataset of Pascal VOC2012 to generate and includes near-focus image, far-focus image, mapping image and all-in-focus image. The experimental result shows that the proposed generative adversarial network model can effectively extract the blurring feature in multi-focus images, and the fused image have good performances on mutual information, phase congruency and structural similarity.
\end{abstract}

Key words: multi-focus image fusion; U-Net; generative adversarial network; loss function

收稿日期: 2020-11-08; 修回日期: 2021-03-19. 基金项目: 国家自然科学基金(61561010); 广西自然科学基金(2017GXNSFAA198089); 广西重点研发计划(桂科 AB18126003, 桂科 AB18221016). 蒋留兵(1973一), 男, 硕士, 研究员, 硕士生导师, 主要研究方向为数字信 息处理; 张点(1995一), 男, 硕士研究生, 论文通讯作者, 主要研究方向为图像融合、模式识别; 潘波(1995一), 男, 硕士研究生, 主要 研究方向为机器学习、姿态识别; 郑朋(1995一), 男, 硕士研究生, 主要研究方向为信号处理、机器学习; 车俐(1977一), 女, 硕士, 高 级实验师, 硕士生导师, 主要研究方向为信号处理. 
由于成像设备景深和光学成像原理的限制, 成像设备获得的图像中的物体不能完全聚焦, 导 致图像中的物体存在模糊. 因此, 研究人员提出了 多聚焦图像融合，以解决上述问题. 多聚焦图像融 合(multi-focus image fusion, MFIF)能够融合同一 场景下一系列具有不同清晰部分的图像，得到一 幅全聚焦图像 ${ }^{[1]}$. 与多聚焦图像相比，全聚焦图像 更加清晰, 含有更多的信息量. 目前, MFIF 主要应 用在机器视觉和目标检测中 ${ }^{[2]}$.

传统的多 MFIF 方法主要分为空间域方法和 转换域方法. 空间域方法将多聚焦图像在像素空 间中融合得到全聚焦图像; 转换域方法将多聚焦 图像转换到其他数域中融合, 融合后进行逆变换 得到全聚焦图像. 空间域方法可以保存多聚焦图 像中的原始像素信息，转换域方法可以提取多聚 焦图像在空间域中隐含的图像的特征. 近年来，随 着 MFIF 的不断发展，空间域方法有逻辑滤波法 ${ }^{[3]}$ 和脉冲耦合神经网络(pulse coupled neural networks, PCNN $)^{[4]}$ 等, 转换域方法有小波变换 ${ }^{[5]}$, Curve Let $^{[5]}$ 和 Contour Let ${ }^{[6]}$ 等方法.

由于独特的生成器和判别器结构, 生成对抗 网络(generative adversarial networks, GAN)的深度 学习模型被应用于图像融合. GAN 模型由生成器 (generator, $G$ )、判别器(discriminator, $D$ ) 和损失 函数 3 部分组成. $G$ 根据给定的源图像生成一幅 真实的融合图像, 以欺骗 $D . D$ 区分 $G$ 生成的融 合图像和真实融合图像. 损失函数确保融合图像 内容不损失. 文献[7]提出一种 GAN 模型融合多曝 光图像. $G$ 由自注意力部分(self-attention)、局部细 节处理部分和融合部分组成. $D$ 由卷积层、全连 接层和 ReLU 层组成. 损失函数由对抗误差、梯 度误差和相似性误差组成. $G$ 中的自注意力部分 和细节处理部分对图像中的特征进行提取, 融合 部分融合提取的特征得到融合后的图像. $D$ 对生 成的融合图像和原始图像进行分辨. 损失函数根 据生成器和融合器的结果保证网络训练结果内容 的完整性. 因此, 融合后的图像更接近真实的融 合图像的概率分布. 文献[8]提出一种 GAN 模型 融合红外和可见光图像. $G$ 使用基于 ResNet 的结 构. D使用基于 VGG11 的结构. 损失函数由内容 损失、细节损失、边缘损失和对抗损失组成. 通 过 GAN 模型融合红外和可见光图像, 损失函数能 够保证融合后的图像分布更加贴近于真实融合图 像的分布.

目前，尽管 GAN 模型已被广泛应用于其他图
像融合场景，但多聚焦图像融合中采用的 GAN 模 型仍然较少. 多聚焦图像融合使用 GAN 模型的主 要难点是多聚焦图像的特征提取、融合图像的分辨 和损失函数的设计. 针对多聚焦图像融合中采用 GAN 模型存在的问题, 本文提出一种在 GAN 中应 用 U-Net 的多聚焦图像融合方法, 分为 $G, D$ 和损 失函数 3 部分, 其中 $G$ 包含特征提取和融合图像 2 个部分. 特征提取采用 U-Net、通道压缩和空间激 励(spatial squeeze and channel excitation, SSE)识别 多聚焦图像中的清晰部分和模糊部分. 融合图像 采用卷积和区域清晰判断的方法得到融合系数图, 并融合图像. 损失函数由 $G$ 的对抗损失、映射损 失、梯度损失、均方误差损失和 $D$ 的对抗损失组 成. 实验结果证明, 本文方法能融合多聚焦图像, 且效果较好.

\section{1 相关工作}

\subsection{GAN}

GAN 是一种估计数据概率分布的深度学习模 型. 在传统方法中, 生成模型通常由已知的概率分 布组成, 如卡方分布和高斯混合分布等, 可以很方 便地执行最大似然函数. 然而, 预定义的分布限制 了生成模型的适应能力 (先验分布越复杂, 似然函 数将越复杂, 越难计算, 对于生成模型的估计将越 困难). GAN 能够估计目标分布并生成与目标分布 相匹配的数据, 而不依赖任何的先验假设 ${ }^{[9]}$. 近年 来, GAN 模型由于其优异的表现，已广泛地应用于 图像融合 ${ }^{[10-11]}$ 和模式识别 ${ }^{[12]}$ 等任务中.

在 GAN 模型中， $G$ 和 $D$ 同时进行训练并形成 博弯进程, 也称为对抗进程. 根据输人的训练集数 据 $x$, 生成器对数据进行采样得到采样数据 $\mathrm{Z}$, 并 根据采样数据的分布生成新的数据, 判别器估计 输人数据为训练集数据的概率. 对抗进程的具体 模型如下: $G$ 从先验分布为 $p_{\mathrm{data}}$ 的训练集数据中 采集数据作为输人, 并且尝试生成不同的样本去 通过 $D$ 的分辨, 使生成样本接近训练数据. $G$ 的 目的是使生成的样本数据尽可能地接近训练集的 数据. $D$ 的目的是分辨输人的数据是 $G$ 生成的数 据还是训练集数据. 对抗模型的博弯过程可以总 结如下: $G$ 不断生成数据去尝试通过 $D$, 生成与 训练数据相似的样本, 增强特征提取能力, $D$ 不 断对数据进行分辨, 以增强自身的分辨能力. 最 终, 根据不同的需要选择 $G$ 或 $D$. 因此, GAN 的数 学模型为 


$$
\begin{aligned}
& \min _{G} \max _{D} V_{\mathrm{GAN}}(G, D)=E_{X \sim p_{\text {data }}(x)}[\lg D(x)]+ \\
& E_{\mathrm{Z} \sim p_{\mathrm{z}}(\mathrm{z})}[\lg (1-D(G(\mathrm{z})))]
\end{aligned}
$$

其中, $E_{x \sim p_{\text {data }}(x)}$ 为按 $p_{\text {data }}(x)$ 的分布对 $x$ 求期望; $z$ 为生成器在训练集数据中的采样数据; $p_{z}(z)$ 为 生成器在训练集数据中进行再采样得到的数据的 分布; $G(z)$ 为生成器根据采样数据生成的结果; $D(x)$ 为训练集数据得到的分辨值; $D(G(x))$ 为生 成器生成的结果得到的分辨值.

\subsection{U-Net}

U-Net 是一种用于图像分割的卷积神经网络 (convolutional neural networks, CNN)方法 ${ }^{[13]}$, 其采用 端到端的编码器-解码器(encoder-decoder)结构, 编 码器进行特征提取, 解码器负责将图像特征恢复到 像素空间. 为了增加对少量数据样本的学习样本, 图像增强中常常采用 U-Net 结构进行数据扩充.

如图 1 所示, U-Net 由 4 个部分组成: 基础结 构 $\left(m_{\mathrm{I}} n_{\mathrm{O}}\right.$ 中, $m_{\mathrm{I}}$ 和 $n_{\mathrm{O}}$ 分别表示结构输入/输出通道 数)、下采样、上采样和长连接. 对于不同的问题, U-Net 基础结构也不同，目的是能够初步提取出输 人图像的视觉特征.下采样的目的是降维、去除图 像中的圥余信息、扩大感知野和保持图像的不变 性. 上采样不仅可以将图像的视觉特征还原到像 素空间中，以观察视觉特征与像素空间中对应模 式的关系, 而且还可以生成较高像素要求的图像. 长连接是通过拼接数据进一步提取数据特征.

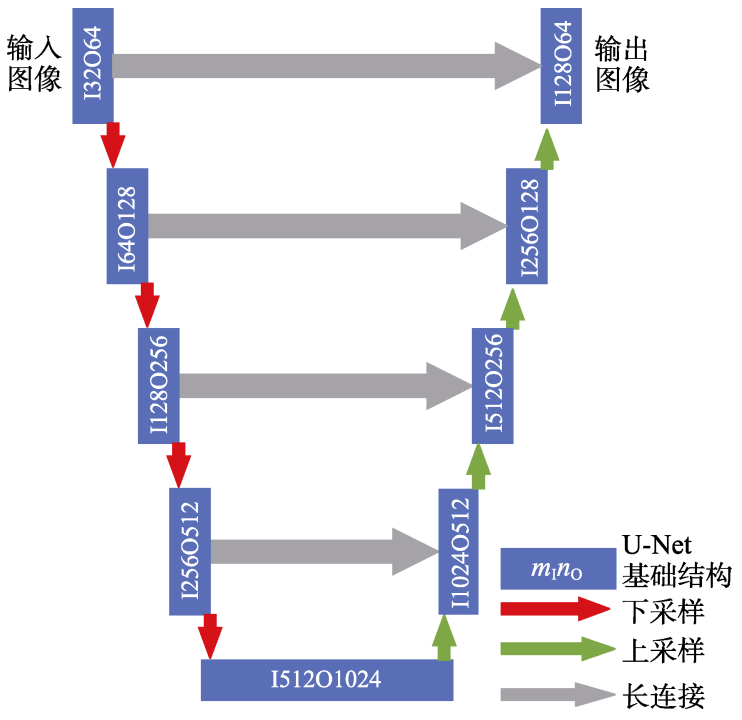

图 1 U-Net 结构

\section{3 注意力机制}

注意力机制源于人能对感官传递的部分信息 进行集中分析而忽略无关信息的行为, 因此, 研究
人员在神经网络中提出对于部分信息进行集中分 析的网络模型, 即注意力机制. 注意力机制是允许 学习模型对语言和视觉等研究任务中的特定信息 进行关注, 从而更有效地完成学习任务的神经网 络方案 ${ }^{[14]}$. 在有限的计算能力下, 注意力机制能 更加有效地分配计算资源, 即注意力机制也是一 种资源分配方案. 注意力机制可以使模型专注于 特定的输人子集, 能够使模型聚焦于数据集中更 关键的信息，降低对其他信息的关注. 因此，注意 力机制可以解决模型在学习过程中产生的信息复 杂性高和圥余度高等缺点, 可以提高模型学习时 的效率和准确性.

文献[15]提出一种信道压缩和激励的架构单 元, 在图像识别和分割方面具有很好的表现. 文献 [16]通过建立通道之间的相互依赖性自适应地校 准通道式的特征响应, 能够有效地提高空间特征 编码. 本文使用通道压缩和空间激励 (spatial squeeze and channel excitation, SSE)的方法增强图 像特征的鲁棒性和泛化性能, 而且在模型中采用 SSE 能学习到更多有意义的空间或通道特征图.

\section{2 本文方法}

\section{1 概要描述}

本文提出的 GAN 方法分为损失函数、 $G$ 和 $D$ 共 3 部分. 其中, 损失函数是为了保证在进行对抗 学习时能够保证融合图像的信息完整性, 减少 $G$ 和 $D$ 对抗学习对于融合图像的影响; $G$ 的目的是 提取多聚焦图像中的特征, 并融合图像; $D$ 的目 的是对生成的全聚焦图像进行分辨. 生成器模型 和判别器模型分别如图 2 和图 3 所示.

\section{2 损失函数}

本文的损失函数共有 2 个部分, 生成器损失 $L_{G}$ 和判别器损失 $L_{D}$. 生成器损失主要包含对抗 损失 $L_{\mathrm{adv}}$ 和内容损失 $L_{\mathrm{con}}$.

$G$ 训练一个从多聚焦到全聚焦图像的映射, 如 近焦图像 $I^{\mathrm{n}}$ 和远焦图像 $I^{\mathrm{d}}$ 到全聚焦图像 $I^{\mathrm{f}}$ 的映射.

为了得到真正的全聚焦图像 $I^{\mathrm{ft}}$ 的概率分布, $G$ 的对抗损失定义为

$$
L_{\mathrm{adv}}=E\left(\lg \left(1-D\left(I^{\mathrm{f}}\right)\right)\right)
$$

其中, $E$ 为期望; $D\left(I^{\mathrm{f}}\right)$ 为 $G$ 生成的融合图像 $I^{\mathrm{f}}$ 通 过 $D$ 得到的值; $L_{\mathrm{adv}}$ 为 $G$ 生成的图像 $I^{\mathrm{f}}$ 保存在 $I^{\mathrm{ft}}$ 中产生的损失. 


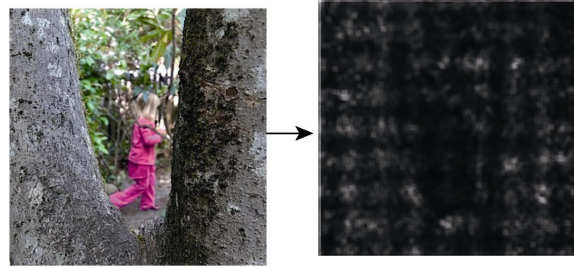

近焦图像

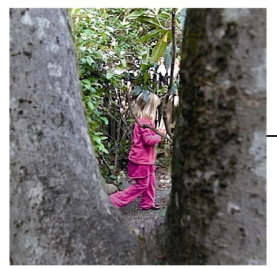

远焦图像

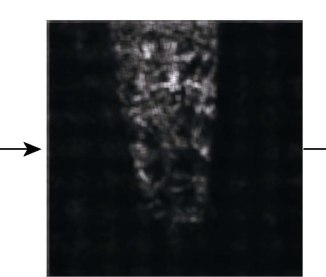

特征提取

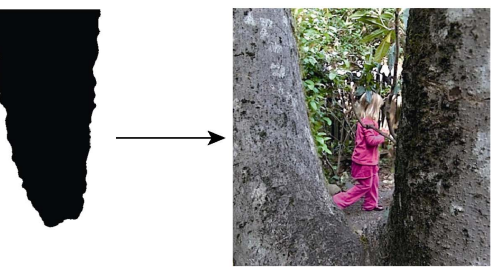

全聚焦图像

图 2 生成器模型

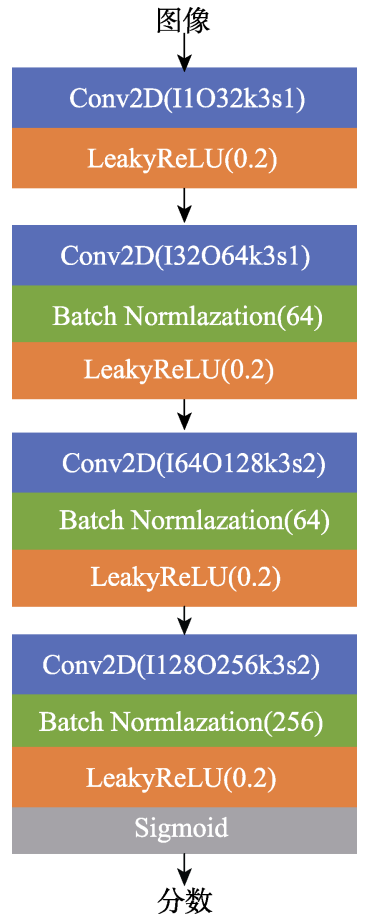

图 3 判别器模型

内容损失 $L_{\text {con }}$ 共有 3 个部分, 分别为映射损失 $L_{\text {map }}$ 、梯度损失 $L_{\text {grad }}$ 和均方误差损失 $L_{\text {mse }}$. 映射损 失 $L_{\mathrm{map}}$ 是指从近焦图像 $I^{\mathrm{n}}$ 和远焦图像 $I^{\mathrm{d}}$ 映射到全 聚焦图像 $I^{\mathrm{f}}$ 的映射图 $I^{\mathrm{map}}$ 和标准映射图 $I^{\mathrm{smap}}$ 的差 异，即

$$
L_{\text {map }}=E\left[\frac{1}{m} \sum_{i=1}^{m} \sum_{j=1}^{n}\left(I_{i, j}^{\text {smap }}-I_{i, j}^{\text {map }}\right)^{2}\right]
$$

其中, $m$ 和 $n$ 分别为图像的长和宽.

梯度损失 $L_{\text {grad }}$ 是指生成的全聚焦图像 $I^{\mathrm{f}}$ 和真 正的全聚焦图像 $I^{\mathrm{ft}}$ 之间的梯度差异, 即

$$
L_{\text {grad }}=E\left[\frac{1}{m n} \sum_{i=1}^{m} \sum_{j=1}^{n}\left|\nabla \Gamma\left(I_{i, j}^{\mathrm{ft}}-\nabla \Gamma I_{i, j}^{\mathrm{f}}\right)\right|\right]
$$

其中, $\nabla$ 为梯度运算; $\Gamma$ 为将图像从 RGB 转换为 灰度图像; $|\cdot|$ 为绝对值操作.

均方误差损失 $L_{\mathrm{mse}}$ 为生成的全聚焦图像 $I^{\mathrm{f}}$ 和 真正的全聚焦图像 $I^{\mathrm{ft}}$ 之间每像素点之间的差的平 方，即

$$
L_{\mathrm{mse}}=E\left[\frac{1}{m n k} \sum_{c=1}^{k} \sum_{i=1}^{m} \sum_{j=1}^{n}\left(I_{i, j, c}^{\mathrm{ft}}-I_{i, j, c}^{\mathrm{f}}\right)^{2}\right]
$$

其中, $k$ 为通道数. 若图像为彩色图像, 则 $k=3$; 若图像为灰度图像, 则 $k=1$.

综上所述, 本文 GAN 方法中的 $L_{\mathrm{adv}}$ 表示生成 器的对抗损失, 即生成器捕获的全聚焦图像 $I^{\mathrm{f}}$ 与 真正的全聚焦图像 $I^{\mathrm{ft}}$ 之间的差异; $L_{\mathrm{map}}$ 为生成 $I^{\mathrm{f}}$ 和 $I^{\mathrm{ft}}$ 之间的映射差异, 即在融合图像中选取的不 同像素值; $L_{\mathrm{grad}}$ 为 $I^{\mathrm{f}}$ 和 $I^{\mathrm{ft}}$ 之间的梯度信息的差 异，此差异主要是代表图像的清晰度和信息量; $L_{\mathrm{mse}}$ 为 $I^{\mathrm{f}}$ 和 $I^{\mathrm{ft}}$ 代表相同位置上像素点之间的差 异. 以 $\lambda$ 为内容损失的系数, 则 $G$ 的损失表达式为

$$
L_{G}=L_{\mathrm{adv}}+\lambda L_{\mathrm{con}}=L_{\mathrm{adv}}+\lambda\left(L_{\mathrm{map}}+L_{\mathrm{grad}}+L_{\mathrm{mse}}\right)
$$

$D$ 用于分辨 $I^{\mathrm{f}}$ 和 $I^{\mathrm{ft}}, D$ 的输出是一个 $0 \sim 1$ 的 数值, 代表输人的图像是 $I^{\mathrm{ft}}$ 的概率, 因此 $D$ 的对抗 损失为

$$
L_{D}=E\left[-\lg \left(1-D\left(I^{\mathrm{f}}\right)\right)\right]+E\left[-\lg \left(D\left(I^{\mathrm{ft}}\right)\right)\right]
$$

\section{3 生成器}

生成器模型的 $G$ 由 2 部分组成, 分别是特征 提取和融合部分. 特征提取和融合的描述分别如 图 4 和图 5 所示. 


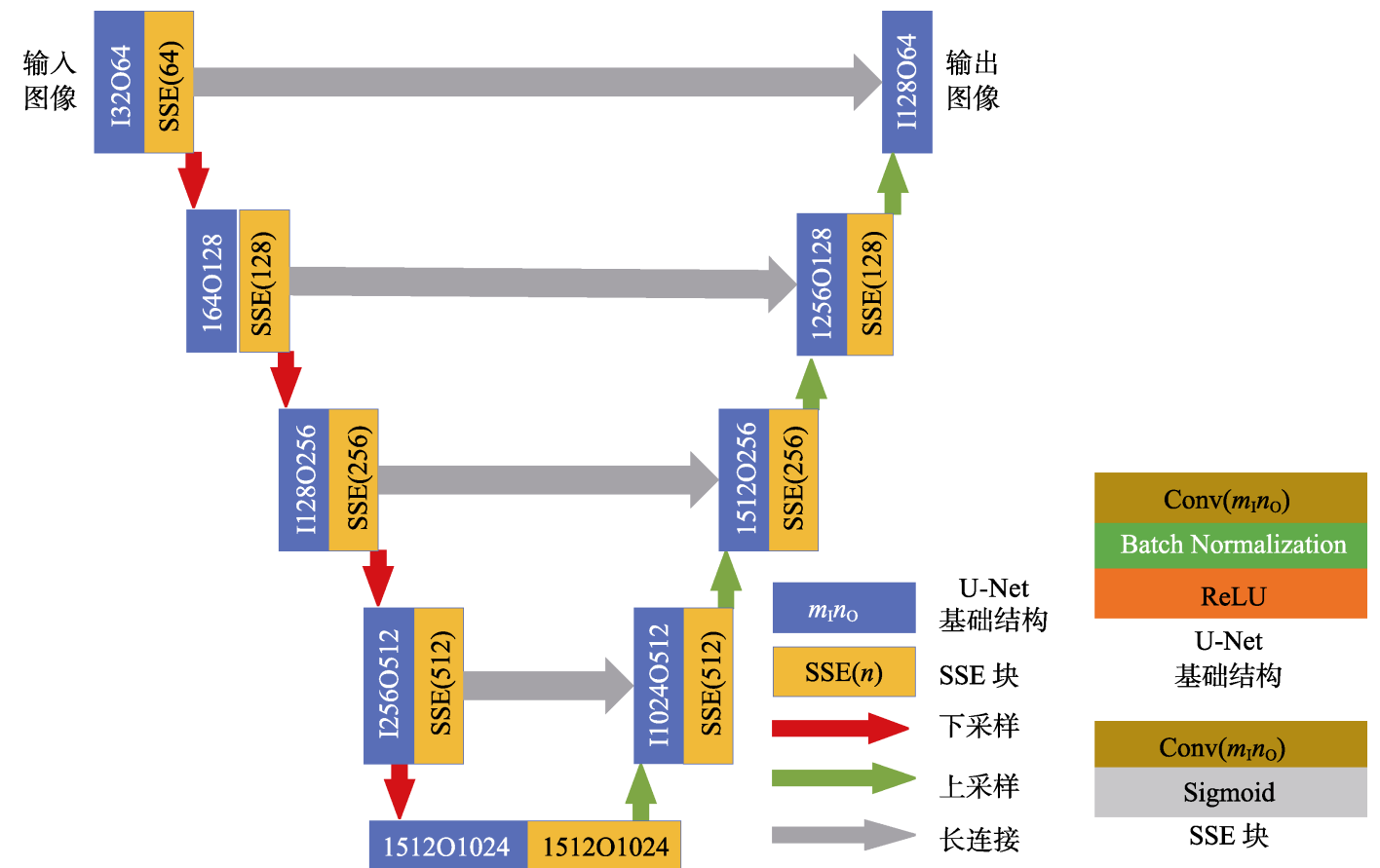

图 4 特征提取

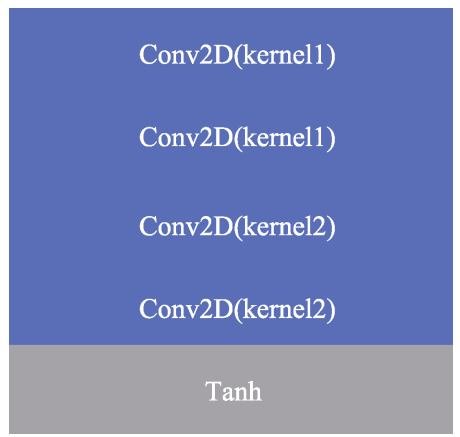

图 5 融合图像

如图 4 所示, U-Net 基础结构结合 SSE 块提取 输人图像的特征. 其中, U-Net 基础结构组成为二 维卷积、块归一化(batch normalization)和 ReLU 激 活层, 图像的上采样由最大池化层 (MaxPool)函数 进行, 图像的下采样由解卷积(ConvTranspose2d) 函数进行, 长连接操作能够保证在进行图像解卷 积操作时更准确地对应图像的细节特征. 在每个 U-Net 基础结构对图像进行处理后, SSE 块再对图 像进行处理能够让输人的图像对其全局的感受野 进行处理，并建立图像与特征通道之间的相关性.

特征提取部分由 U-Net 基础结构和 SSE 块组成. U-Net 结构能提取深层次的图像特征, SSE 块可以对 图像中的特征部分给予关注, 使 U-Net 在后续的处 理中对图像特征给予更高的关注. 由于多聚焦图像 存在部分模糊且模糊部分互补和有限的细节信息的 特征, 本文提出一种 U-Net 和 SSE 块相结合的多聚
焦图像特征提取方法. 特征提取结合 U-Net 独特的 编码器逐渐减小图像的空间尺寸, 解码器对图像中 物体的细节和空间尺寸进行恢复, 并且对应的编码 器和解码器之间存在直接的连接, 能够使神经网络 更准确地恢复与提取图像中的特征. SSE 块能够将空 间通道进行压缩，压缩后得到的实数具有全局的感 受野, 并且输出的维度与输人的特征通道相匹配. 因此, 特征通道的全局分布与压缩后的实数存在函 数关系, 而且输人的层也可以获取到全局的感受野. SSE 块能够通过参数为每个特征通道生成权重, 参 数用来显示建立与特征通道之间的相关性. 综上, 本文所采用的 U-Net 结构能够准确地判别多聚焦图 像的清晰特征. 如图 2 所示, 通过特征提取部分得到 多聚焦图像的特征，通过融合图像部分得到图像的 融合系数图, 最后得到多聚焦图像.

由 U-Net 结构分别获取图像的特征部分, 将其 拼接作为融合部分的输人部分. 融合部分由采用不 同卷积核的二维卷积层 $k_{1}=[0,0,0 ; 1,0,0 ; 0,0,0]$ 和 $k_{1}=[0,1,0 ; 0,0,0 ; 0,0,0]$ 组成, 采用不同卷积核的目 的是提取不同方向的图像特征. 由于池化操作会 减少图像的特征并且在图像融合中会造成图像模 糊，在融合部分中本文并没有使用池化层. 最后, 采用 Tanh 函数得到多聚焦图像的融合系数图, 根 据融合系数图融合多聚焦图像.

\section{4 判别器}

由于判别器的任务是区分图像, 因此判别器 
的结构比生成器简单许多. 如图 3 所示, 本文的判 别器总共有 4 个卷积层, 卷积层后有块归一化或 LeakyReLU 函数, 或者两者都有. 在前 2 层中, 卷 积层的步长被设置为 1 , 在后 2 层中卷积层的步长 被设置为 2. 第 2 4 层的每个卷积层后都有块归一 化. 第 4 个卷积层的输出由 Sigmoid 函数激活, Sigmoid 函数能够得到输人图像是真正的全聚焦图 像 $I^{\mathrm{ft}}$ 的概率.

\section{3 实验及结果分析}

\section{1 实验数据集}

本文采用的训练数据集是 Pascal VOC2012 $2^{[17]}$ 中用于图像分割的数据集. 由于多聚焦图像可以 认为是图像中的某一部分被点扩散函数进行模糊 后得到的, 本文采用人工模糊的方法生成 GAN 模 型中的多聚焦图像训练集. 本文的点扩散函数采 用高斯函数. 由于在 Pascal VOC2012 图像中含有 分割轮廓图, 可以得到图像中的物体或人, 因此, 本文利用原始图像与分割轮廓图 GAN 的训练集, 分割轮廓图采用二分类的分割轮廓图. 首先, 生成 方差为 5 , 尺寸为 $10 \times 10$ 的高斯核滤波 $l$; 然后, 将 $l$ 分别于分割轮廓图中的 2 类进行卷积, 即

$$
\begin{gathered}
n=(i \times m) \otimes l \\
f=(i \times(1-m)) \otimes l
\end{gathered}
$$

其中, $i$ 为 VOC2012 数据集的原始数据; $m$ 为分 割轮廓图; $l$ 为高斯滤波器; $n$ 和 $f$ 分别为生成的 近焦和远焦图像. 实验所用的部分训练集图像如 图 6 所示.

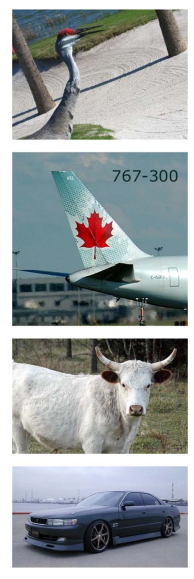

a. 原始图像
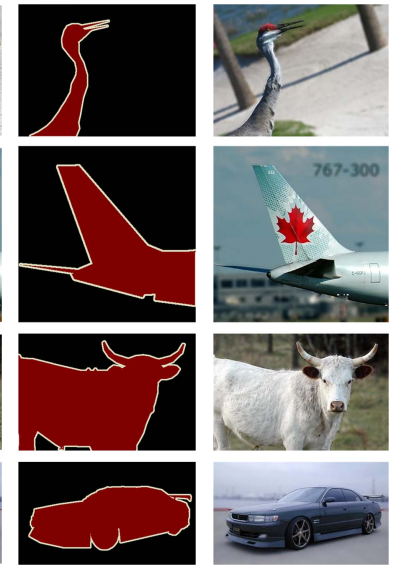

b. 分割图

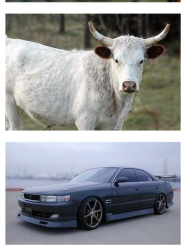

c. 近焦图像

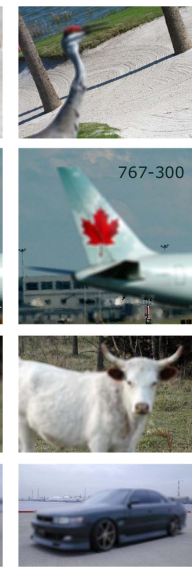

d. 远焦图像
图 6 训练集图像

本文采用的数据集来自文献[18-19]以及一些 应用于已发表论文中的多聚焦图像, 共 50 幅图像,
包括彩色图像和灰度图像。文献[18]使用尼康 D5000 相机创建多聚焦图像数据集, 包括 27 对图 像. 文献[19]中包含了 20 对 512×512 的多聚焦彩色 图像. 选取不同文献中的图像用于测试能够验证 本文方法对不同的成像设备的实用性. 选取的测 试图像中的聚焦部分的形状各不相同, 能够验证 本文方法对于不同的聚焦形状的性能.

\section{2 结果及分析}

为了对本文方法进行评价, 采用如图 7 所示的 测试图像 ${ }^{[17-18]}$ 进行验证, 并与文献[20-24]中的实 验方法进行比较. 文献[20]提出一种基于自适应分 块的小波变换方法进行图像融合(简称 DWTDE); 文献[21]提出一种使用深度卷积神经网络的 MFIF 方法(简称 CNN); 文献[22]提出一种梯度和联通区 域判断的 MFIF 方法(简称 GCF); 文献[23]提出一 种非监督学习神经网络的 MFIF 方法 (简称 U2FUSION); 文献[24]提出一种多规模梯度权重 的 MFIF 方法(简称 MWGF). 为了验证本文方法的 实验结果, 而且由于单一客观评价标准无法准确 评价融合后的全聚焦图像的质量, 因此采用互信 息 (mutual information, MI) 、边缘信息保留度 $\left(Q^{A B / F}\right)^{[25]}$ 、信息熵(entropy, $E$ )、机器视觉的评价标 准 $\left(Q_{\mathrm{CB}}\right)^{[26]}$ 、相位一致性 (phase congruency, $\left.Q_{\mathrm{PC}}\right)^{[27]}$ 、结构相似性(structural similarity, $\left.Q_{\mathrm{SS}}\right)^{[28]}$ 和感知相似性 $\left(Q_{\mathrm{LP}}\right)^{[29]}$ 作为评价指标. 由于本文采 用的 MFIF 有 2 幅原始图像, 因此本文使用 2 幅源 图像分别对融合图像求其 $Q_{\mathrm{LP}}$, 然后进行相加; 由 于 $Q_{\mathrm{LP}}$ 只能对彩色图像进行评价, 因此对于 U2FUSION 实验结果未能进行评价.

实验对比结果如图 7 图 13 所示, 融合结果中 存在差异的位置用红色矩形标出. 从图 7 图 13 中 可以看出, DWTDE 方法融合后的图像具有较好的 彩色效果, 但是对于图像中的一些模糊像素点的 位置存在误判, 导致融合后的图像存在不清晰部 分; CNN 方法能够对多聚焦图像中的清晰部分进 行较精准的判断, 但在融合边缘存在畸变; GCF 方 法能够对多聚焦图像中的模糊部分进行较为精准的 判断, 但在图像的融合边缘上出现了视觉伪影; U2FUSION 方法获得的图像存在细节损失，导致图 像中的一些细节部分的观察不清晰; MWGF 方法 尽管对一些多聚焦图像能够实现较精准的融合, 但对一些多聚焦图像的聚焦区域存在误判，泛化 性能较差. 总体而言, 本文方法所获得的融合结果 具有较好的视觉效果, 且细节信息能够进行保留, 融合图像的边缘处没有出现伪影和畸变等现象. 


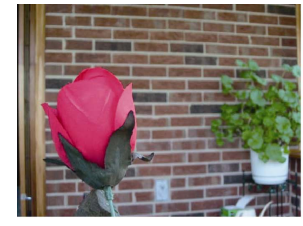

Flower1

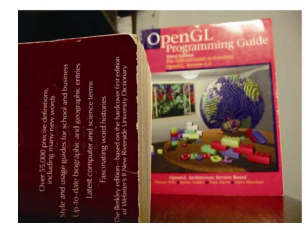

Book1

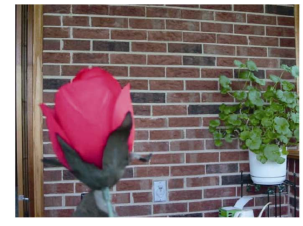

Flower2

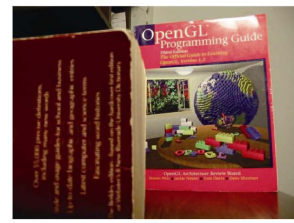

Book2

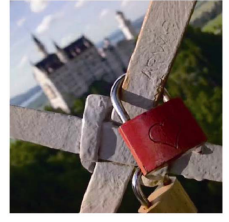

Lock1

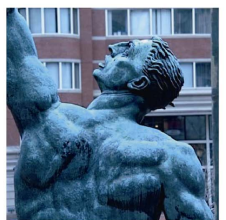

Sculpture1

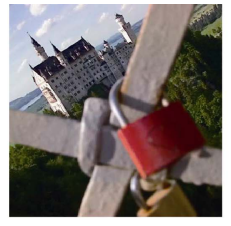

Lock2

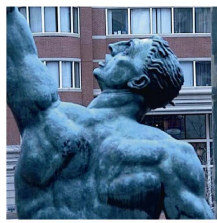

Sculpture2

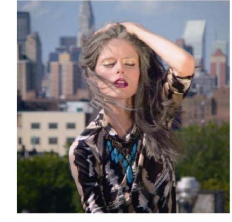

Lady1

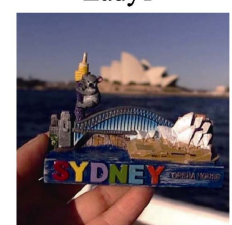

Sydney 1

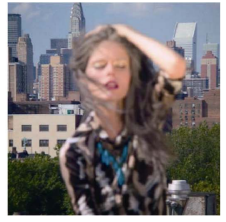

Lady2

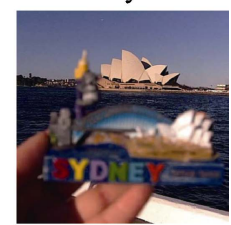

Sydney2

图 7 测试集图像

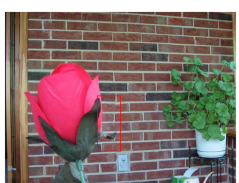

a. DWTDE ${ }^{[20]}$

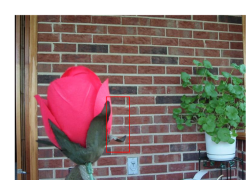

b. $\mathrm{CNN}^{[21]}$

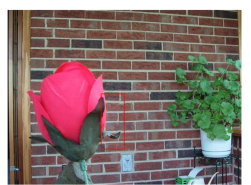

c. $\mathrm{GCF}^{[22]}$

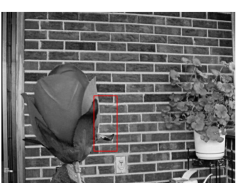

d. U2FUSION ${ }^{[23]}$

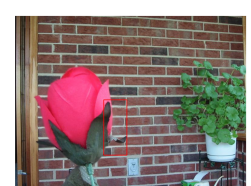

e. $\mathrm{MWGF}^{[24]}$

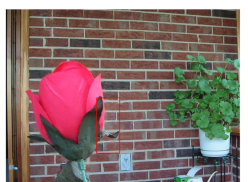

f. 本文

图 8 不同方法对 Flower 图像融合结果

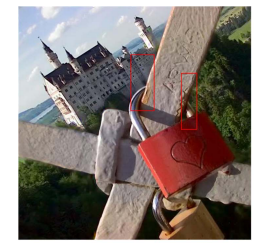

a. DWTDE ${ }^{[20]}$

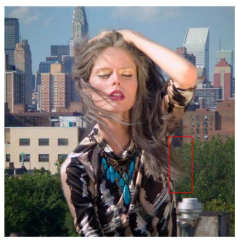

a. DWTDE ${ }^{[20]}$

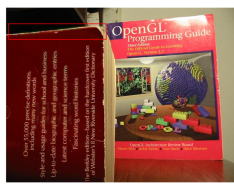

a. DWTDE ${ }^{[20]}$

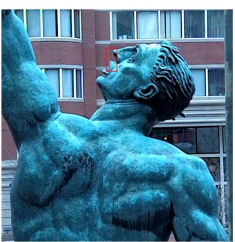

a. DWTDE ${ }^{[20]}$

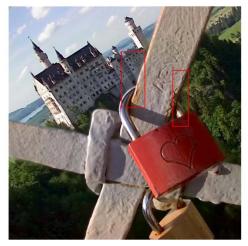

b. $\mathrm{CNN}^{[21]}$

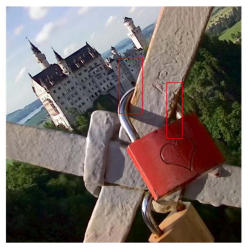

c. $\mathrm{GCF}^{[22]}$

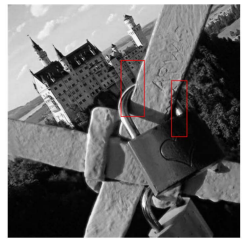

d. U2FUSION ${ }^{[23]}$

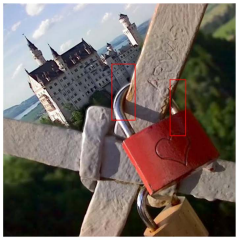

e. $\mathrm{MWGF}^{[24]}$

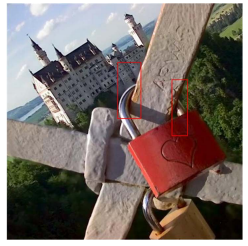

f. 本文

图 9 不同方法对 Lock 图像融合结果

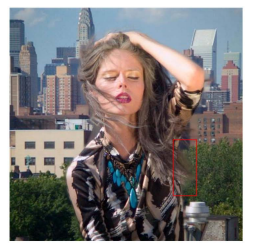

b. $\mathrm{CNN}^{[21]}$

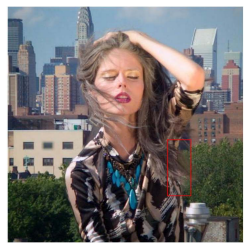

c. $\mathrm{GCF}^{[22]}$

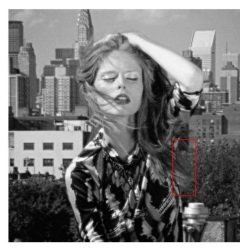

d. U2FUSION ${ }^{[23]}$

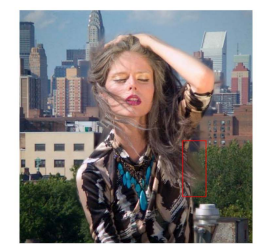

e. $\mathrm{MWGF}^{[24]}$

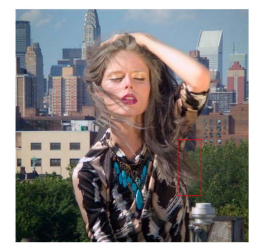

f. 本文

图 10 不同方法对 Lady 图像融合结果

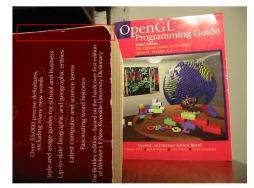

b. $\mathrm{CNN}^{[21]}$

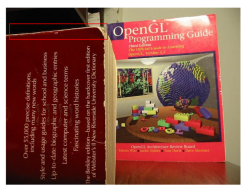

c. $\mathrm{GCF}^{[22]}$

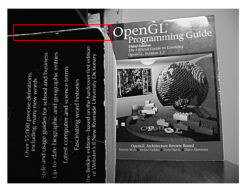

d. U2FUSION ${ }^{[23]}$

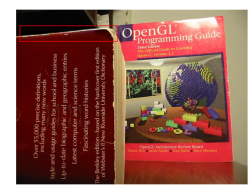

e. $\mathrm{MWGF}^{[24]}$

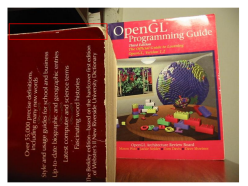

f. 本文

图 11 不同方法对 Book 图像融合结果

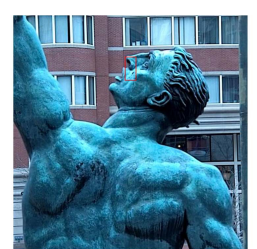

b. $\mathrm{CNN}^{[21]}$

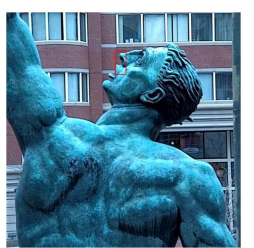

c. $\mathrm{GCF}^{[22]}$

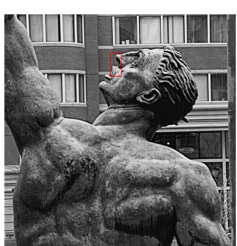

d. U2FUSION ${ }^{[23]}$

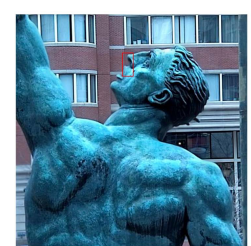

e. $\mathrm{MWGF}^{[24]}$

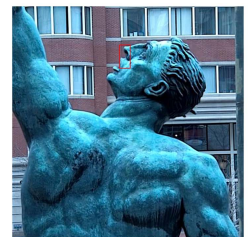

f. 本文

图 12 不同方法对 Sculpture 图像融合结果 


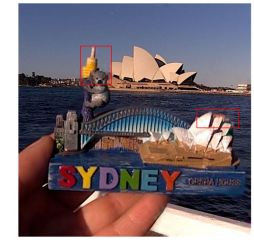

a. DWTDE ${ }^{[20]}$

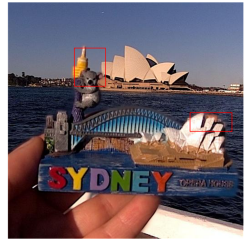

b. $\mathrm{CNN}^{[21]}$

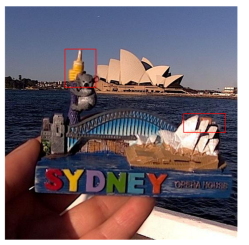

c. $\mathrm{GCF}^{[22]}$

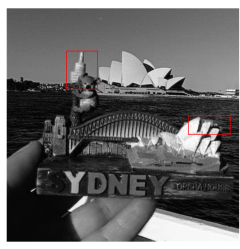

d. U2FUSION ${ }^{[23]}$

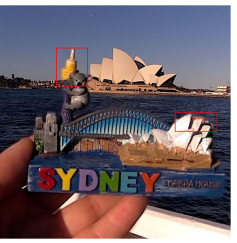

e. $\mathrm{MWGF}^{[24]}$

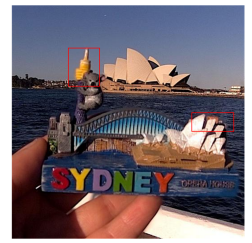

f. 本文

图 13 不同方法对 Sydney 图像融合结果

实验结果表明，本文方法可以有效地提取多聚焦 图像中清晰部分的特征, 获得较好的视觉效果.

由于人的视觉感知对融合图像中较为细小的 差异无法进行判别, 本文通过多种客观评价标准 对不同融合方法所获得的融合图像质量进行分析, 比较结果如表 1 表 6 所示, 从表 1、表 2 和表 6 中 可以观察到, 本文方法的 $Q_{\mathrm{SS}}$ 略低于 $\mathrm{CNN}$ 方法, MI, $Q^{A B / F}, E, Q_{\mathrm{CB}}$ 和 $Q_{\mathrm{PC}}$ 值均大于其他融合方 法. 从表 3 中可以观察到, U2FUSION 方法的 $E$ 和 $\mathrm{GCF}$ 方法的 $Q_{\mathrm{CB}}$ 均大于本文方法, 但结合图 8 可以 看出, U2FUSION 方法和 GCF 方法的融合边缘存 在伪影和模糊, 而本文方法融合图像的边缘存在 部分细节损失导致 $E$ 和 $Q_{\mathrm{CB}}$ 小于 U2FUSION 和 GCF 方法. 从表 4 中可以观察到, CNN 方法的 $Q_{\mathrm{CB}}$
大于本文方法, 但观察图 9 可知, 本文方法的融合 边缘和细节部分比 CNN 方法更加清晰. 表 1 表 5 中本文方法 $Q_{\mathrm{LP}}$ 都大于其他方法, 表明本文方法在 感知性能上具有更加优异的表现. 此外, 表 7 展示 了不同方法所获得的融合图像的客观指标的平均 值, 可以看出本文方法 $E$ 指标低于其他方法, 但其 他各项指标上均大于其他对比方法. 通过以上分 析可以认为, 本文方法在客观指标上的表现优于 其他方法.

综合以上主观评价和客观评价及分析, 可以得 出, 本文方法能够较好地提取多聚焦图像中的模糊 部分特征, 并且对图像进行较为细致的融合. 实验 结果证明, 本文方法具有较好的视觉效果和客观评 价指标，而且整体的融合结果优于其他融合方法.

表 1 不同方法对 Flower 融合结果的客观评价

\begin{tabular}{|c|c|c|c|c|c|c|c|}
\hline 方法 & MI & $Q^{A B / F}$ & $E$ & $Q_{\mathrm{CB}}$ & $Q_{\mathrm{PC}}$ & $Q_{\mathrm{SS}}$ & $Q_{\mathrm{LP}}$ \\
\hline DWTDE $^{[20]}$ & 2.6090 & 0.6808 & 7.1661 & 0.7898 & 0.9617 & 0.7749 & 0.2506 \\
\hline $\mathrm{CNN}^{[21]}$ & 2.8532 & 0.7048 & 7.1763 & 0.8252 & 0.9825 & 0.7938 & 0.2464 \\
\hline $\mathrm{GCF}^{[22]}$ & 2.4649 & 0.6028 & 7.1822 & 0.7924 & 0.8999 & 0.6881 & 0.2705 \\
\hline $\mathrm{U}^{\mathrm{FUSION}}{ }^{[23]}$ & 2.3242 & 0.5946 & 7.4557 & 0.5935 & 0.8634 & 0.7556 & \\
\hline $\mathrm{MWGF}^{[24]}$ & 2.7554 & 0.6980 & 7.1792 & 0.8153 & 0.9769 & 0.7893 & 0.2507 \\
\hline 本文 & 2.8731 & 0.7187 & 7.4976 & 0.8263 & 0.9892 & 0.7874 & 0.2712 \\
\hline
\end{tabular}

注. 粗体代表当前评价标准最优结果.

表 2 不同方法对 Lock 融合结果的客观评价

\begin{tabular}{lccccccc}
\hline \multicolumn{1}{c}{ 方法 } & $\mathrm{MI}$ & $Q^{A B / F}$ & $E$ & $Q_{\mathrm{CB}}$ & $Q_{\mathrm{PC}}$ & $Q_{\mathrm{SS}}$ & $Q_{\mathrm{LP}}$ \\
\hline DWTDE $^{[20]}$ & 2.7482 & 0.7119 & 7.7154 & 0.7771 & 0.9654 & 0.8260 & 0.4896 \\
$\mathrm{CNN}^{[21]}$ & 2.8312 & 0.7417 & 7.7164 & 0.8041 & 0.9860 & $\mathbf{0 . 8 6 1 ~ 1}$ & 0.4814 \\
GCF $^{[22]}$ & 2.8461 & 0.7374 & 7.7099 & 0.7970 & 0.9849 & 0.8487 & 0.4856 \\
U2FUSION $^{[23]}$ & 2.5241 & 0.6499 & 7.4957 & 0.6228 & 0.8068 & 0.7574 & 0.7964 \\
MWGF $^{[24]}$ & 2.8529 & 0.6665 & 7.7114 & 0.7517 & 0.9855 & 0.4834 \\
本文 & $\mathbf{2 . 8 7 4 ~ 7}$ & $\mathbf{0 . 7 4 2 ~ 1}$ & $\mathbf{7 . 7 1 8 ~ 4}$ & $\mathbf{0 . 8 0 8 ~ 6}$ & $\mathbf{0 . 9 8 9 5}$ & 0.8489 & $\mathbf{0 . 4 8 9 ~ 9}$ \\
\hline
\end{tabular}

注. 粗体代表当前评价标准最优结果. 
表 3 不同方法对 Lady 融合结果客观评价

\begin{tabular}{lccccccc}
\hline \multicolumn{1}{c}{ 方法 } & $\mathrm{MI}$ & $Q^{\mathrm{AB} / F}$ & $E$ & $Q_{\mathrm{CB}}$ & $Q_{\mathrm{PC}}$ & $Q_{\mathrm{SS}}$ & $Q_{\mathrm{LP}}$ \\
\hline DWTDE $^{[20]}$ & 2.7049 & 0.6999 & 7.7084 & 0.7770 & 0.9670 & 0.8726 & 0.4290 \\
$\mathrm{CNN}^{[21]}$ & 2.7647 & $\mathbf{0 . 7 1 8 9}$ & 7.7086 & 0.7956 & 0.9858 & 0.8928 & 0.4271 \\
GCF $^{[22]}$ & 2.7808 & 0.7146 & 7.7093 & $\mathbf{0 . 8 0 3 2}$ & 0.9815 & 0.8857 & 0.4309 \\
U2FUSION $^{[23]}$ & 2.4847 & 0.6262 & $\mathbf{7 . 7 5 7 2}$ & 0.6066 & 0.8671 & 0.7764 & \\
MWGF $^{[24]}$ & 2.7009 & 0.7124 & 7.7142 & 0.7811 & 0.9757 & 0.8833 & 0.4301 \\
本文 & $\mathbf{2 . 8 0 5 3}$ & $\mathbf{0 . 7 1 8 ~ 9}$ & 7.7100 & 0.7997 & $\mathbf{0 . 9 8 9 ~ 1}$ & $\mathbf{0 . 8 9 7 2}$ & $\mathbf{0 . 4 4 1 4}$ \\
\hline
\end{tabular}

注. 粗体代表当前评价标准最优结果.

表 4 不同方法对 Book 融合结果客观评价

\begin{tabular}{lccccccc}
\hline \multicolumn{1}{c}{ 方法 } & $\mathrm{MI}$ & $Q^{\mathrm{AB} / F}$ & $E$ & $Q_{\mathrm{CB}}$ & $Q_{\mathrm{PC}}$ & $Q_{\mathrm{SS}}$ \\
\hline DWTDE $^{[20]}$ & 2.6890 & 0.6611 & 7.3064 & 0.7452 & 0.8906 & 0.8493 \\
$\mathrm{CNN}^{[21]}$ & 2.9377 & 0.7263 & 7.2549 & $\mathbf{0 . 8 0 7 7}$ & 0.9871 & 0.8935 \\
GCF $^{[22]}$ & 2.8555 & 0.7193 & 7.2559 & 0.7782 & 0.9750 & 0.8808 \\
U2FUSION $^{[23]}$ & 2.4703 & 0.5996 & 6.8826 & 0.5066 & 0.6955 & 0.2596 \\
MWGF $^{[24]}$ & 2.8580 & 0.7234 & 7.2973 & 0.7866 & 0.9601 & 0.8885 \\
本文 & $\mathbf{2 . 9 4 4 6}$ & $\mathbf{0 . 7 2 8 ~ 3}$ & $\mathbf{7 . 3 4 9 7}$ & 0.7927 & $\mathbf{0 . 9 8 9 ~ \mathbf { 0 }}$ & $\mathbf{0 . 8 9 9 3}$ \\
\hline
\end{tabular}

注. 粗体代表当前评价标准最优结果.

表 5 不同方法对 Sculpture 融合结果客观评价

\begin{tabular}{|c|c|c|c|c|c|c|c|}
\hline 方法 & MI & $Q^{A B / F}$ & $E$ & $Q_{\mathrm{CB}}$ & $Q_{\mathrm{PC}}$ & $Q_{\mathrm{SS}}$ & $Q_{\mathrm{LP}}$ \\
\hline DWTDE $^{[20]}$ & 2.6930 & 0.7593 & 7.6808 & 0.8295 & 0.9645 & 0.8961 & 0.2645 \\
\hline $\mathrm{CNN}^{[21]}$ & 2.7780 & 0.7845 & 7.6838 & 0.8524 & 0.9780 & 0.9123 & 0.2664 \\
\hline $\mathrm{GCF}^{[22]}$ & 2.7610 & 0.7747 & 7.6849 & 0.8464 & 0.9747 & 0.9049 & 0.2697 \\
\hline $\mathrm{U}^{2} \mathrm{FUSION}^{[23]}$ & 2.4226 & 0.6840 & 7.6702 & 0.6454 & 0.9079 & 0.7994 & \\
\hline MWGF $^{[24]}$ & 2.6985 & 0.7655 & 7.6785 & 0.8266 & 0.9719 & 0.9014 & 0.2760 \\
\hline 本文 & 2.8283 & 0.7888 & 7.6869 & 0.8537 & 0.9789 & 0.9186 & 0.2765 \\
\hline
\end{tabular}

注. 粗体代表当前评价标准最优结果.

表 6 不同方法对 Sydney 融合结果客观评价

\begin{tabular}{lccccccc}
\hline \multicolumn{1}{c}{ 方法 } & $\mathrm{MI}$ & $Q^{A B / F}$ & $E$ & $Q_{\mathrm{CB}}$ & $Q_{\mathrm{PC}}$ & $Q_{\mathrm{SS}}$ & $Q_{\mathrm{LP}}$ \\
\hline DWTDE $^{[20]}$ & 2.7023 & 0.7278 & 7.5608 & 0.7359 & 0.9571 & 0.8002 & $\mathbf{0 . 4 6 1 ~ 6}$ \\
$\mathrm{CNN}^{[21]}$ & 2.7817 & 0.7728 & 7.5646 & 0.7887 & 0.9896 & $\mathbf{0 . 8 4 4 \mathbf { 1 }}$ & 0.4555 \\
$\mathrm{GCF}^{[22]}$ & 2.7859 & 0.7721 & 7.5653 & 0.7876 & 0.9900 & 0.8381 & 0.4583 \\
U2FUSION $^{[23]}$ & 2.5019 & 0.6390 & 7.4741 & 0.6032 & 0.8481 & 0.7125 & \\
MWGF $^{[24]}$ & 2.7391 & 0.7676 & 7.5660 & 0.7780 & 0.9848 & 0.8379 & 0.4559 \\
本文 & $\mathbf{2 . 8 3 2 ~ 6}$ & $\mathbf{0 . 7 7 6 ~ 9}$ & $\mathbf{7 . 5 6 6 ~ 3}$ & $\mathbf{0 . 7 9 3 ~ 4}$ & $\mathbf{0 . 9 9 1 ~ 0}$ & 0.8393 & 0.4579 \\
\hline
\end{tabular}

注. 粗体代表当前评价标准最优结果.

表 7 不同方法对融合图像质量指标对比(平均值)

\begin{tabular}{|c|c|c|c|c|c|c|c|}
\hline 方法 & MI & $Q^{A B / F}$ & $E$ & $Q_{\mathrm{CB}}$ & $Q_{\mathrm{PC}}$ & $Q_{\mathrm{SS}}$ & $Q_{\mathrm{LP}}$ \\
\hline DWTDE $^{[20]}$ & 2.6993 & 0.7129 & 7.3809 & 0.7663 & 0.9500 & 0.7891 & 0.4463 \\
\hline $\mathrm{CNN}^{[21]}$ & 2.8324 & 0.7524 & 7.3262 & 0.7978 & 0.9857 & 0.8346 & 0.4276 \\
\hline $\mathrm{GCF}^{[22]}$ & 2.6949 & 0.6648 & 7.4268 & 0.7370 & 0.9184 & 0.7322 & 0.4476 \\
\hline $\mathrm{U}^{2} \mathrm{FUSION}^{[23]}$ & 2.4595 & 0.6304 & 7.4376 & 0.6169 & 0.8515 & 0.6963 & \\
\hline MWGF $^{[24]}$ & 2.7745 & 0.7378 & 7.3628 & 0.7805 & 0.9779 & 0.8211 & 0.4312 \\
\hline 本文 & 2.8712 & 0.7598 & 7.3104 & 0.7981 & 0.9872 & 0.8358 & 0.4512 \\
\hline
\end{tabular}

注. 粗体代表当前评价标准最优结果. 
为了对本文方法的时间效率进行对比, 将所 有方法运行在 Windows10, Intel i5 CPU 的计算机 中. 将文献[18-19]中的图像作为测试图像计算量化 性能, 即将各方法在每对多聚焦图像中花费的平均 时间作为量化性能进行比较, 结果如表 8 所示.

表 8 不同融合方法时间效率比较

\begin{tabular}{lr}
\hline \multicolumn{1}{c}{ 方法 } & 时间/s \\
\hline DWTGE $^{[20]}$ & 38.14 \\
CNN $^{[21]}$ & 336.07 \\
GCF $^{[22]}$ & 44.10 \\
U2FUSION $^{[23]}$ & 33.73 \\
MWGF $^{[24]}$ & 11.20 \\
本文 & 28.27 \\
\hline
\end{tabular}

从表 8 可以看出, MWGF 方法消耗时间比较 少, 原因是它采用了多规模分解和图像结构显著 性的融合方法. DWTDE 方法消耗时间主要由小波 变换和采用自适应分块所产生. GCF 方法由于在深 度学习模型中采用梯度信息和连通区域分析的方 法, 消耗时间较长. U2FUSION 方法中采用了一种 端到端的神经网络框架进行融合, 其中采用 VGG 网络进行融合提取, 因此其消耗时间较少. CNN 方 法采用了卷积神经网络，但过多的时间用于判别 是否图像聚焦, 导致其耗费时间较多. 本文方法由 于采用 U-Net 和注意力机制, 导致在耗费时间上没 有最好的表现．虽然本文方法时间效率没有达到 最佳, 但是在大多数融合图像的质量表现优于其 他方法.

\section{4 结 语}

本文提出一种基于 GAN 和 U-Net 的 MFIF 方 法, 本文方法的主要优势是生成器中提取特征采 用了 U-Net 和 SSE 块. 由于采用 U-Net 结构的网络 结合 SSE 块能够扩大网络对多聚焦图像特征捕获 的感受野, 因此生成器能更加准确地识别多聚焦 图像中的清晰部分和模糊部分的特征, 使融合后 的图像能够保留源图像的细节信息和纹理信息. 本文采用 Pascal VOC2012 数据集生成所需要的训 练集, 由于采用监督学习的方法, 本文方法能够更 加有效地识别多聚焦图像中清晰部分的特征.从 视觉效果上看, 本文方法获得的融合图像清晰且 含有丰富的细节信息; 从客观评价指标上看，本文 方法获得的图像指标总体优于其他方法, 说明本 文方法是一种有效且可行的 MFIF 方法.
综上所述, 本文提出的 GAN 模型是一种能够 用于 MFIF 且能够取得较好实验结果的实验方法. 随着在图像融合方面的深人研究, 未来在 MFIF 方 面的主要研究方向如下.

(1) 深度学习的高效图像融合模型. 目前, 尽 管深度学习方法和硬件设备的不断发展, 使图像 融合能在较短的时间内完成, 但深度学习方法很 难有数量级运行时间的缩短. 这主要是因为深度 学习理论的复杂性和各种复杂的深度学习模型的 提出, 深度学习方法运行时间数量级上的缩短主 要依赖于更加简单的深度学习模型的提出.

（2）精准的图像评价标准. 由于目前不存在一 种绝对精确的图像清晰度的评价, 每种图像评价 标准都有其优劣势, 不能对每幅图像都有一种较 为准确的评价, 因此在图像融合结果的评价中只 能采用多种客观评价标准复合的方式评价图像.

\section{参考文献(References):}

[1] Song Ruixia, Wang Meng, Wang Xiaochun. A multi-focus image fusion algorithm combining NSCT with edge detection[J]. Journal of Computer-Aided Design \& Computer Graphics, 2016, 28(12): 2134-2141(in Chinese)

(宋瑞霞, 王孟, 王小春. NSCT 与边缘检测相结合的多聚焦 图像融合算法 [J]. 计算机辅助设计与图形学学报, 2016, 28(12): 2134-2141)

[2] Li S T, Kang X D, Fang L Y, et al. Pixel-level image fusion: a survey of the state of the art[J]. Information Fusion, 2017, 33: 100-112

[3] Wang Z B, Chen L N, Li J, et al. Multi-focus image fusion with random walks and guided filters[J]. Multimedia Systems, 2019, 25(4): 323-335

[4] Du C B, Gao S S. Multi-focus image fusion algorithm based on pulse coupled neural networks and modified decision map[J]. Optik, 2018, 157: 1003-1015

[5] Li S T, Yang B. Multi-focus image fusion by combining curvelet and wavelet transform[J]. Pattern Recognition Letters, 2008, 29(9): 1295-1301

[6] Anandhi D, Valli S. An algorithm for multi-sensor image fusion using maximum a posteriori and nonsubsampled contourlet transform[J]. Computers \& Electrical Engineering, 2018, 65: 139-152

[7] Xu H, Ma J Y Zhang X P. MEF-GAN: multi-exposure image fusion via generative adversarial networks[J]. IEEE Transactions on Image Processing, 2020, 29: 7203-7216

[8] Ma J Y, Liang P W, Yu W, et al. Infrared and visible image fusion via detail preserving adversarial learning[J]. Information Fusion, 2020, 54: 85-98

[9] Ian G, Jean P A, Mehdi M, et al. Generative adversarial nets[C] //Proceedings of the 27th International Conference on Neural Information Processing Systems. Cambridge: MIT Press, 2014: 2672-2680

[10] Ma J Y, Yu W, Liang P W, et al. FusionGAN: a generative ad- 
versarial network for infrared and visible image fusion[J]. Information Fusion, 2019, 48: 11-26

[11] Zhang H, Le Z L, Shao Z F, et al. MFF-GAN: an unsupervised generative adversarial network with adaptive and gradient joint constraints for multi-focus image fusion[J]. Information $\mathrm{Fu}-$ sion, 2021, 66: 40-53

[12] $\mathrm{Xu}$ Zhijing, Wang Dong. Multi-pose recognition with two-cycle generative adversarial network[J]. Acta Optica Sinica, 2020, 40(19): 63-72(in Chinese) (徐志京, 王东. 基于双路循环生成对抗网络的多姿态人脸 识别方法[J]. 光学学报, 2020, 40(19): 63-72)

[13] Ronneberger O, Fischer P, Brox T. U-Net: convolutional networks for biomedical image segmentation[C] //Proceedings of the 18th International Conference on Medical Image Computing and Computer-Assisted Intervention. Heidelberg: Springer, 2015: 234-241

[14] Adam K. The attention mechanisms in neural network[J]. Robot Industry, 2017(6): 12-17(in Chinese)

(Adam K. 神经网络中的注意力机制 [J]. 机器人产业, 2017(6): 12-17)

[15] Hu J, Shen L, Albanie S, et al. Squeeze-and-excitation networks[J]. IEEE Transactions on Pattern Analysis and Machine Intelligence, 2020, 42: 2011-2023

[16] Roy A G, Navab N, Wachinger C. Concurrent spatial and channel squeeze and excitation in fully convolutional networks[C] //Proceedings of International Conference on Medical Image Computing and Computer-Assisted Intervention. Heidelberg: Springer, 2018: 421-429

[17] Everingham M, Eslami S M A, van Gool L, et al. The PASCAL visual object classes challenge: a retrospective[J]. International Journal of Computer Vision, 2015, 111(1): 98-136

[18] Nejati M, Samavi S, Shirani S. Multi-focus image fusion using dictionary based sparse representation[J]. Information Fusion, 2015, 25: 72-84

[19] Slavica S, Zdenka B. Multifocus image fusion based on the first level of empirical mode decomposition[C] //Proceedings of the 19th International Conference on Systems, Signals and
Image Processing. Los Alamitos: IEEE Computer Society Press, 2012: 604-607

[20] Liu Yu, Wang Zengfu. Multi-focus image fusion based on wavelet transform and adaptive block[J]. Journal of Image and Graphics, 2013, 18(11): 1435-1444(in Chinese)

(刘羽, 汪增福. 结合小波变换和自适应分块的多聚焦图像 快速融合 [J]. 中国图象图形学报, 2013, 18(11): 1435-1444)

[21] Yu L, Xun C, Hu P, et al. Multi-focus image fusion with a deep convolutional neural network[J]. Information Fusion, 2017, 36: 191-207

[22] Xu H, Fan F, Zhang H, et al. A deep model for multi-focus image fusion based on gradients and connected regions[J]. IEEE Access, 2020, 8: 26316-26327

[23] Xu H, Ma J Y, Jiang J J, et al. U2Fusion: a unified unsupervised image fusion network[J]. IEEE Transactions on Pattern Analysis and Machine Intelligence (Early Access), DOI: 10.1109/TPAMI.2020.3012548

[24] Zhou Z Q, Li S, Wang B. Multi-scale weighted gradient-based fusion for multi-focus images[J]. Information Fusion, 2014, 20 : 60-72

[25] Xydeas C S, Petrovic V. Objective image fusion performance measure[J]. Electronics Letters, 2000, 36(4): 308-309

[26] Chen H, Pramod K V. A human perception inspired quality metric for image fusion based on regional information[J]. Information Fusion, 2007, 8(2): 193-207

[27] Zhao J Y, Robert L, Liu Z. Performance assessment of combinative pixel-level image fusion based on an absolute feature measurement[J]. International Journal of Innovative Computing Information and Control, 2006, 3(6): 1433-1447

[28] Yang C, Zhang J Q, Wang X R, et al. A novel similarity based quality metric for image fusion[J]. Information Fusion, 2018, 9(2): 156-160

[29] Zhang R, Isola P, Efros A A, et al. The unreasonable effectiveness of deep features as a perceptual metric[C] //Proceedings of the IEEE/CVF Conference on Computer Vision and Pattern Recognition. Los Alamitos: IEEE Computer Society Press, 2018: 586-595 